\title{
Extrinsic and Intrinsic Stability of Formamidinium Lead Bromide Perovskite Solar Cells Yielding High Photovoltage
}

\author{
Neha Arora, ${ }^{1, \#}$ M. Ibrahim Dar, ${ }^{1, ~ \# ~ M o j t a b a ~ A b d i-J a l e b i, ~}{ }^{2}$ Fabrizio Giordano, ${ }^{1}$ Norman Pellet, ${ }^{1}$ Gwénolé \\ Jacopin, ${ }^{3}$ Richard H. Friend, ${ }^{2}$ Shaik Mohammed Zakeeruddin, ${ }^{1}$ Michael Grätzel ${ }^{1}$ \\ ${ }^{1}$ Laboratory of Photonics and Interfaces, Institute of Chemical Sciences and Engineering, École Polytechnique \\ Fedérale de Lausanne, CH-1015 Lausanne, Switzerland. \\ ${ }^{2}$ Cavendish Laboratory, Department of Physics, University of Cambridge, JJ Thomson Avenue, Cambridge, CB3 \\ 0HE, United Kingdom. \\ ${ }^{3}$ Laboratory of Quantum Optoelectronics, Institute of Physics, École Polytechnique Fedérale de Lausanne, CH-1015 \\ Lausanne, Switzerland.
}

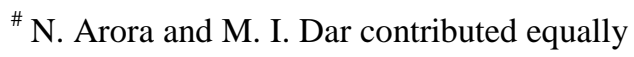




\begin{abstract}
Extrinsic as well as intrinsic instability of perovskite solar cells has proved to be an invincible bottleneck towards their commercialization. The extrinsic instability, which involves the affinity of perovskite material towards moisture could be overcome by encapsulation while the intrinsic stability can be achieved by identifying the right chemical composition of light absorber. Herein, we report on both extrinsic as well as intrinsic stability of formamidinium lead bromide $\left[\mathrm{CH}\left(\mathrm{NH}_{2}\right)_{2} \mathrm{PbBr}_{3}=\mathrm{FAPbBr}_{3}\right]$ perovskite solar cells yielding a high photovoltage. The fabrication of $\mathrm{FAPbBr}_{3}$ devices displaying an outstanding photovoltage of $1.53 \mathrm{~V}$ and a power conversion efficiency of over $8 \%$ was realized by modifying the mesoporous $\mathrm{TiO}_{2} / \mathrm{FAPbBr}_{3}$ interface using lithium treatment. The reasons for improved photovoltaic performance were unfolded by a combination of techniques including photothermal deflection absorption spectroscopy (PDS), transient photovoltage and charge extraction analysis, and time-integrated and time-resolved photoluminescence. With lithium treated $\mathrm{TiO}_{2}$ films, the studies based on PDS reveal that the $\mathrm{TiO}_{2}-\mathrm{FAPbBr}_{3}$ interface exhibits less energetic disorder, and the emission dynamics showed that electron injection from the conduction band of $\mathrm{FAPbBr}_{3}$ into that of mesoporous $\mathrm{TiO}_{2}$ is faster than for the untreated scaffold. Moreover, compared to the device with pristine $\mathrm{TiO}_{2}$, the charge carrier recombination rate within a device based on lithium treated $\mathrm{TiO}_{2}$ film is one order of magnitude lower. Importantly, the operational stability of perovskites solar cells examined under maximum power point revealed that the $\mathrm{FAPbBr}_{3}$ material is intrinsically (under nitrogen) as well as extrinsically (in ambience) stable, as the unsealed devices retained over $95 \%$ of the initial efficiency under continuous illumination for $150 \mathrm{~h}$ in nitrogen, dry air as well as in $60 \%$ humidity $\left(\mathrm{T}=\sim 60{ }^{\circ} \mathrm{C}\right.$ ). The demonstration of high photovoltage, a record for $\mathrm{FAPbBr}_{3}$ together with robust stability renders our work of practical significance.
\end{abstract}




\section{Introduction}

The salient optoelectronic properties, such as high extinction coefficient, long charge carrier diffusion lengths, ambipolar charge transport exhibited by organometal halide perovskites make them an impeccable light harnessing material for photovoltaics. ${ }^{1,2}$ Following the first demonstration by Miyasaka and coworkers, ${ }^{3}$ the transition in the application of organometal halide perovskites as a light harvester in liquid dye-sensitized solar cell to solid state hybrid solar cells has been revolutionary.

Organometal halide perovskites can be broadly based on either mesoscopic or planar heterojunction cell structure, which in turn defines the interface between the perovskite material and electron transporting materials (ETMs). Although there are many reports pertaining to molecular engineering of hole-transporting materials (HTMs), the mesoporous electron transporting materials (ETMs) for perovskite solar cells have been comparatively less explored. The first report on dye sensitized solar cells (DSSCs) involving mesoscopic $\mathrm{TiO}_{2}$ films triggered enormous interest towards employing anatase $\mathrm{TiO}_{2}$ as an electron selective contact. ${ }^{4}$ The position of conduction band, appropriate band gap, optoelectronic characteristics and favorable intrinsic photoelectric property render $\mathrm{TiO}_{2}$ as an inevitable electron transporting and hole blocking layer in solar cells. ${ }^{5,6}$ Furthermore, the engineering of the band gap and electron transporting properties of $\mathrm{TiO}_{2}$ by anionic or cationic doping using $\mathrm{Y}^{3+}, \mathrm{Nb}^{5+}$, and $\mathrm{Ga}^{3+}$ has been intensively investigated especially in DSSCs and more recently in perovskite solar cells (PSCs). ${ }^{7,8,9}$ Majorly, such judicious doping allows the modification of optoelectronic properties by reducing the electronic trap states in $\mathrm{TiO}_{2}$. This in turn prolongs the electron lifetime and eventually improves the charge carrier collection efficiency.

An impressive advancement in the efficiencies of PSCs over the years has been realized by a plethora of research work on tuning the device architecture, optimization of fabrication process, and material engineering. ${ }^{10,11,12}$ Thus far, most of the efforts have been devoted towards the development of perovskite solar cells revealing high short-circuit current densities, whereas the fabrication of devices yielding high photovoltage has been explored scantly. Bromide-based perovskite possessing higher band gap is of particular interest for use in tandem and in driving uphill electrochemical reactions such as the photoreduction of $\mathrm{CO}_{2}$ using water as an electron source which demand high voltages. ${ }^{13}$ Previously, the $\mathrm{CH}_{3} \mathrm{NH}_{3} \mathrm{PbBr}_{3}$ solar cells with high open- 
circuit voltage have been realized by optimization of chemical composition of perovskite material, control of crystallization process, or by energy level engineering of hole extracting layer. ${ }^{14,15,16}$ However, $\mathrm{CH}_{3} \mathrm{NH}_{3} \mathrm{PbBr}_{3}$ contains a highly polar organic cation (DM= 2.29D) which shows strong affinity towards moisture and polar solvent. ${ }^{17}$ Owing to better photostability, $\mathrm{CsPbBr}_{3}$ yielding a moderate open-circuit voltage of $1.25 \mathrm{~V}$ was proposed as an alternative to $\mathrm{CH}_{3} \mathrm{NH}_{3} \mathrm{PbBr}_{3}{ }^{18}$

Herein, we report on the fabrication of efficient and stable formamidinium lead bromide perovskite solar cell using modified $\mathrm{TiO}_{2}$ and spiro-OMeTAD, respectively, as electron and hole transporting materials. We demonstrate the effect of surface modification of mesoporous $\mathrm{TiO}_{2}$ photoanode by lithium on the performance of $\mathrm{FAPbBr}_{3}$ perovskite solar cells. Recently, Heo et al. and Giordano et al. reported improvement in the electronic properties of Li-treated mesoscopic $\mathrm{TiO}_{2} \cdot{ }^{19,20}$ Both the studies focused on the bulk properties of mesoporous $\mathrm{TiO}_{2}$, however, this study specifically involves the effect of lithium on the interfacial characteristics, which considerably dictated the overall performance of the $\mathrm{FAPbBr}_{3}$ devices. A combination of techniques, including photothermal deflection absorption spectroscopy, time-integrated and timeresolved photoluminescence and transient analysis was employed to understand energetic disorder of $\mathrm{TiO}_{2}$ /perovskite interface, charge injection from absorber into charge extraction layers and charge carrier recombination rate within a device, respectively. Interestingly, $\mathrm{FAPbBr}_{3}$ PSCs displayed an impressive open circuit photovoltage (Voc) of $1.53 \mathrm{~V}$ with a power conversion efficiency exceeding 8\% under simulated AM 1.5G illumination. More importantly, at a maximum power point, un-encapsulation devices showed extraordinary stability not merely in ambient air but also under humid conditions (> 60\%). As is known, the instability of perovskite solar cells could significantly hamper their long term device operation, therefore, demonstration of such a robust stability of $\mathrm{FAPbBr}_{3}$ devices yielding outstanding photovoltage renders our work unprecedented and of high significance.

\section{Results and discussion}

\section{Fabrication of $\mathrm{FAPbBr}_{3}$ perovskite solar cells}

The lithium ( $\mathrm{Li}$ ) treatment of the mesoporous $\mathrm{TiO}_{2}$ was carried out via spin coating a solution of LiTFSI dissolved in acetonitrile solvent as previously described. ${ }^{21}$ Subsequently, $\mathrm{FAPbBr}_{3}$ 


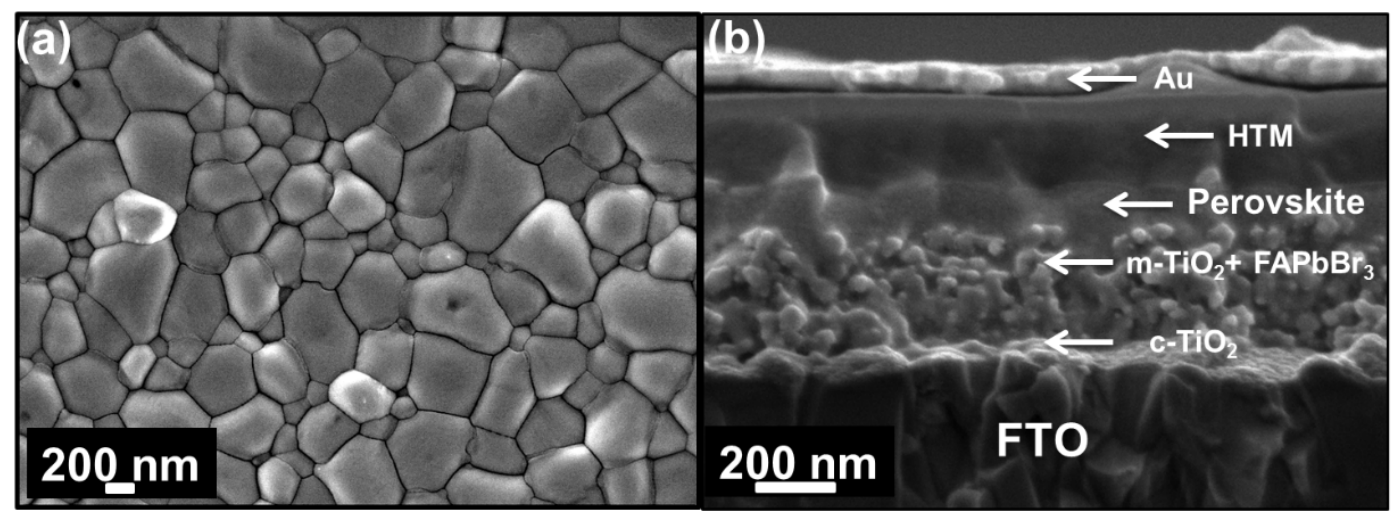

Figure 1. Morphological analysis. a. High-magnification SEM top view image of the $\mathrm{FAPbBr}_{3}$ film and b. Cross-sectional SEM image of a complete $\mathrm{FAPbBr}_{3}$ perovskite device.

perovskite films were deposited by two-step deposition procedure using $\mathrm{PbBr}_{2}$ and $\mathrm{CH}\left(\mathrm{NH}_{2}\right)_{2} \mathrm{Br}$ $(\mathrm{FABr}) .^{22}$ [see Methods Section]. The X-ray diffraction pattern (Figure S1) acquired from the resulting perovskite film could be indexed to the cubic phase of $\mathrm{FAPbBr}_{3}$ perovskite and the surface morphology probed by scanning electron microscopy (SEM) revealed the formation of uniform and dense films with full surface coverage (Figure 1a). Figure 1b shows the crosssection SEM image of the device with a configuration FTO/compact $\mathrm{TiO}_{2} /$ meso$\mathrm{TiO}_{2} / \mathrm{FAPbBr}_{3} / \mathrm{Spiro-OMeTAD} / \mathrm{Au}$ and the total thickness of the device is $\sim 600 \mathrm{~nm}$.

\section{Photovoltaic performance of $\mathrm{FAPbBr}_{3}$ perovskite solar cells}

To understand the impact of Li-treatment on the overall performance, we fabricated devices using pristine and Li-treated mesoporous- $\mathrm{TiO}_{2}$. The photocurrent density-voltage $(\mathrm{J}-\mathrm{V})$ curves measured under AM 1.5 global (AM 1.5G) illumination are shown in Figure 2a. The champion $\mathrm{FAPbBr}_{3}$ device based on pristine mesoporous $\mathrm{TiO}_{2}$ showed an open-circuit voltage $\left(V_{\text {oc }}\right)$ of 1.44 $\mathrm{V}$ and a power-conversion efficiency (PCE) of 6.95\%.$^{23}$ However, the best $\mathrm{FAPbBr}_{3}$ device involving Li-treated meso- $\mathrm{TiO}_{2}$ device displayed an outstanding $V_{\text {ос }}$ of $1.53 \mathrm{~V}$ with impressive PCE of over $8 \%$. To the best of our knowledge, this is the highest efficiency, and a record $V_{\text {oc }}$ reported for $\mathrm{FAPbBr}_{3}$ devices. The increase in the photovoltaic performance upon Li-treatment of $\mathrm{TiO}_{2}$ was confirmed by measuring device parameters for a batch of 20 devices (Figure 2b). We noted a remarkably higher average $V_{\text {oc }}$ value for the devices involving Li-treated mesoporous $\mathrm{TiO}_{2}$ in comparison to pristine $\mathrm{TiO}_{2}(1.516 \pm 0.009$ vs $1.42 \pm 0.016)$ resulting in higher average 

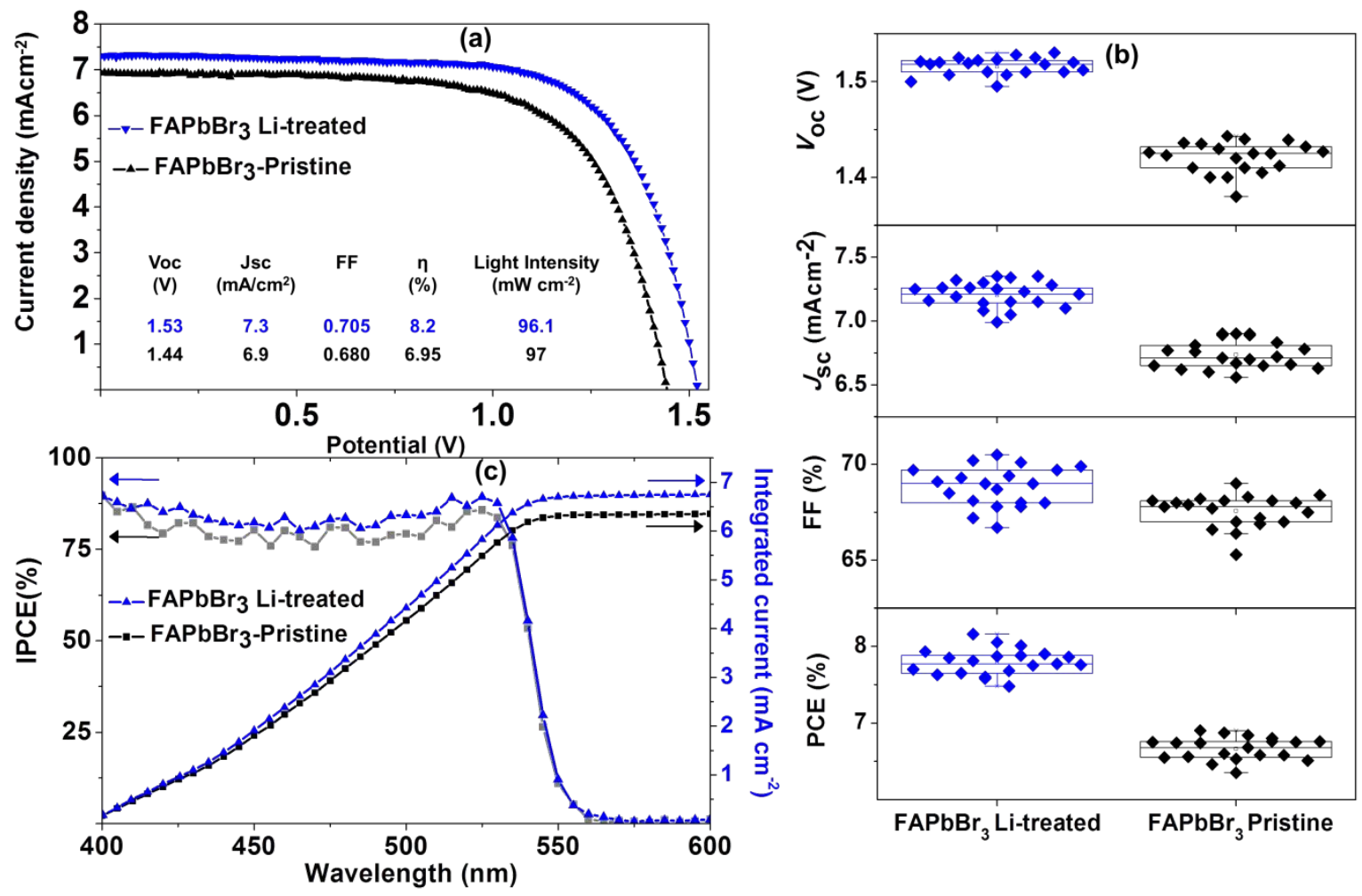

Figure 2. Photovoltaic characterization of $\mathrm{FAPbBr}_{3}$ perovskite devices based on pristine and Litreated $\mathrm{TiO}_{2}$. a. Current density-voltage (J-V) curves for the champion $\mathrm{FAPbBr}_{3}$ solar cells collected under AM1.5 simulated sunlight recorded at a scan rate of $0.1 \mathrm{~V} \mathrm{~s}^{-1}$. b. Statistics (J-V metrics) for 20 different $\mathrm{FAPbBr}_{3}$ devices based on Li-treated and pristine $\mathrm{TiO}_{2}$. c. IPCE spectra as a function of the wavelength of monochromatic light and integrated $J_{\mathrm{sc}}$ values calculated from the corresponding IPCE spectra recorded under the standard AM 1.5 solar emission.

PCE $(7.81 \pm 0.17$ vs $6.7 \pm 0.15)$ with slight improvement in $J_{\text {sc }}(7.25 \pm 0.12$ vs $6.73 \pm 0.10)$ and FF $(68.8 \pm 1.05$ vs $67.5 \pm 0.91)$. All the devices exhibited a negligible hysteresis in the $\mathrm{J}-\mathrm{V}$ curves during reverse and forward scans (Figure S2).

The incident photon-to-current-efficiency (IPCE) or external quantum efficiency (EQE) spectra for devices involving pristine and Li-treated mesoporous $\mathrm{TiO}_{2}$ are shown in Figure 2c. In the IPCE spectrum, the onset of the photocurrent is $~ 550 \mathrm{~nm}$, which is in agreement with the band gap of $\mathrm{FAPbBr}_{3}$ perovskite material $(2.26 \mathrm{eV}) .{ }^{24}$ We observe efficient charge extraction between 400-550 nm with a maximum EQE of 85\% and the photocurrent densities $\left(J_{\mathrm{sc}}\right)$ integrated from IPCE spectra are slightly lower that the $J_{\mathrm{sc}}$ values extracted from the J-V curves. 


\section{Charge extraction and photovoltage transient analysis}

A striking difference between the PCE arises mainly from the relatively large open circuit voltage ( $\sim 90 \mathrm{mV}$ ) exhibited by devices based on Li-treated photoanode. To unravel the causes for high photovoltage, we performed charge extraction and photovoltage transient measurements (Figure 3). Mostly, charge extraction reveals a shift in the conduction band position or modifications in the density of states (DOS) of the electron transporting layer. The electronic properties in mesoporous materials are in fact governed by a trapping-detrapping of charges within the conduction band and sub-bandgap states. Moreover, the transport and recombination processes are extremely sensitive to the distance between the quasi Fermi energy level of the trapped electrons and the conduction band of the semiconductor. In Figure 3a the plot of the extracted charge at $V_{\text {oc }}$ versus the open circuit voltage shows that under high illumination intensity, for a given voltage the device employing untreated $\mathrm{TiO}_{2}$ stores up to twice the charge compared to the device based on Li-treated electrode. In addition, we observed a beneficial change in the shape of the two curves, rather than a simple shift. Furthermore, the modification of the density of states shows a dramatic effect on the electron lifetime, which is almost one order of magnitude more for the Li-treated $\mathrm{TiO}_{2}$ than the pristine one (Figure 3b). Arguably, such a prolonged electron lifetime in combination with the passivation of the sub-bandgap states in the $\mathrm{TiO}_{2}$ could give rise to the enhanced photovoltage.
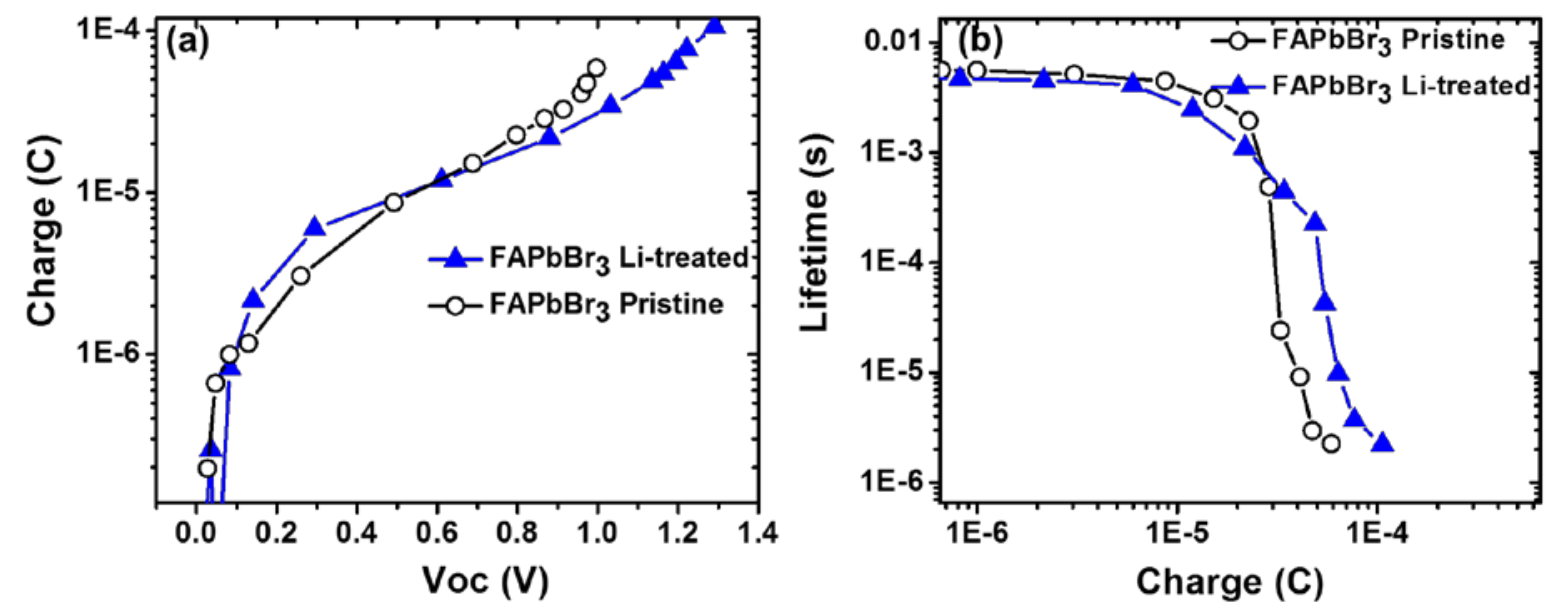

Figure 3. Charge extraction and photovoltage transient analysis. a. Total charge extracted for open circuit voltage versus open circuit voltage for different light intensities and $\mathbf{b}$. Electron lifetime versus the extracted charge at different light intensities. 


\section{Photothermal deflection spectroscopy (PDS)}

To probe the sub-bandgap optical absorption and the density of states near the band edge of mesoporous $\mathrm{TiO}_{2}$ and $\mathrm{FAPbBr}_{3}$ films, photothermal deflection absorption spectroscopy (PDS) measurements were performed (Figure 4). It is evident that the Li-treated mesoporous $\mathrm{TiO}_{2}$ has a significant lower sub-bandgap absorption level compared to the pristine one (Figure 4a). In addition, the density of states is considerably shifted towards the band edge of $\mathrm{TiO}_{2}$ upon adding lithium to the system. The sub-bandgap absorption tail has an exponential variation, $A \sim \exp (E$ $\left./ E_{\mathrm{u}}\right)$, defining the Urbach Energy $\left(E_{\mathrm{u}}\right)$, which is a measure for the degree of disorder within the material. ${ }^{25}$ The inset in Figure 4a shows a decrease in $E_{\mathrm{u}}$ from $360 \mathrm{meV}$ to $155 \mathrm{meV}$ upon Litreatment, which further confirms that $\mathrm{Li}$ reduces the number of trap states (respective fitting errors are shown in Figure S3, S4).

To further analyze whether the Li-treatment of mesoporous $\mathrm{TiO}_{2}$ has any influence on the subbandgap optical absorption of $\mathrm{FAPbBr}_{3}$, we recorded the PDS absorption spectra of $\mathrm{FAPbBr}_{3}$ deposited on pristine and Li-treated mesoporous $\mathrm{TiO}_{2}$ (Figure $\mathbf{4 b}$ ). In both samples, a sharp band edge at $2.17 \mathrm{eV}$ close to the bandgap of $\mathrm{FAPbBr}_{3}$ is observed. Furthermore, the comparative analysis of PDS absorption spectra shows that the sub-bandgap absorption of $\mathrm{FAPbBr}_{3}$
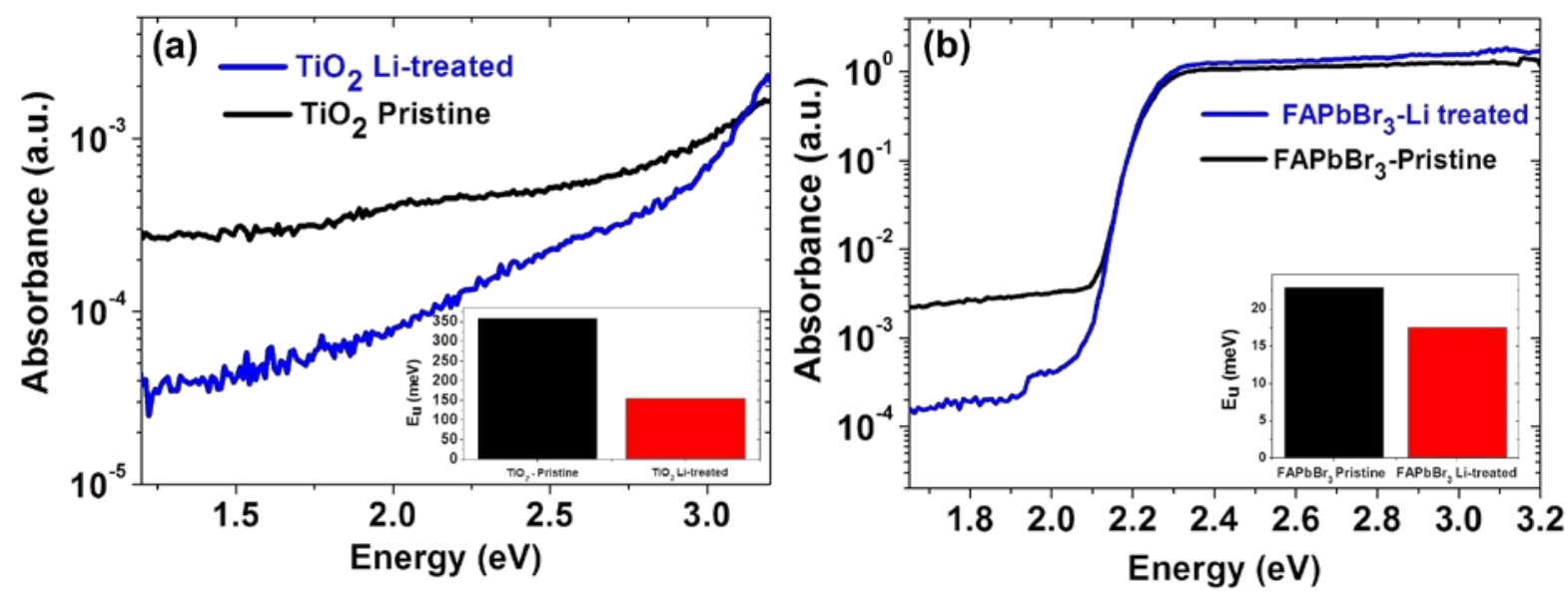

Figure 4. The absorbance spectra of pristine and Li-treated $\mathrm{mp}-\mathrm{TiO}_{2}$ and $\mathrm{FAPbBr}_{3}$ films deposited on pristine and Li-treated $\mathrm{TiO}_{2}$ measured using the photothermal deflection spectroscopy technique. (a) Absorbance spectra of pristine (3a, black curve) and Li-treated (3a, blue curve) $\mathrm{mp}^{-\mathrm{TiO}_{2}}$ and (b) $\mathrm{FAPbBr}_{3}$ deposited on pristine (3b, black curve), and Li-treated (3b, blue curve) $\mathrm{mp}-\mathrm{TiO}_{2}$. The inset shows the corresponding Urbach energies. 
deposited onto $\mathrm{Li}$-treated $\mathrm{TiO}_{2}$ drops by an order of magnitude. In addition, the $E_{\mathrm{u}}$ decreases from $24 \mathrm{meV}$ to $18 \mathrm{meV}$, which implies that the $\mathrm{FAPbBr}_{3}$ deposited onto Li-treated $\mathrm{TiO}_{2}$ exhibits a lower level of energetic disorder (Figure $\mathbf{4 b}$, inset). Therefore, from PDS studies, we surmise that lithium not only pacifies the surface traps in mesoporous $\mathrm{TiO}_{2}$, but also suppresses the sub-bandgap states present at the interface the $\mathrm{FAPbBr}_{3}$ film, which is also confirmed from charge extraction measurements. We further envisage that such a low level of interfacial disorder could bring down the parasitic recombination of charge carriers and enhance the Voc of the device.

\section{Photoluminescence}

The emission and charge carrier dynamics in $\mathrm{FAPbBr}_{3}$ films, and across perovskite- $\mathrm{TiO}_{2}$ and perovskite-spiro-OMeTAD interfaces were examined using time-integrated and time-resolved photoluminescence spectroscopy. Figure 5a depicts the time-integrated photoluminescence (TIPL) of $\mathrm{FAPbBr}_{3}$ deposited onto mesoporous $\mathrm{Al}_{2} \mathrm{O}_{3}$ layer (with and without spiro-OMeTAD), and onto pristine and Li-treated mesoporous $\mathrm{TiO}_{2}$ substrates. $\mathrm{FAPbBr}_{3}$ deposited on electronically inactive $\mathrm{Al}_{2} \mathrm{O}_{3}$ mesoporous layer exhibits a strong and narrow emission around $2.27 \mathrm{eV}$. Expectedly, in the presence of charge extraction layers, i.e., $\mathrm{TiO}_{2}$ and spiro-OMeTAD, the intensity of PL is quenched considerably, from which we infer rapid charge transfer occurring across interfaces between absorber and charge extraction layers. To understand the nature of charge carrier dynamics in different samples more quantitatively, we recorded timeresolved photoluminescence (TRPL) (Figure 5b). The life time of charge carriers was estimated by fitting the PL decay traces using two-exponential decay model as summarized in Table 1. It is evident that charge carrier recombination is quite slow ( $\tau_{1}=41 \mathrm{~ns}$ ) in $\mathrm{FAPbBr}_{3}$ film deposited on mesoporous $\mathrm{Al}_{2} \mathrm{O}_{3}$, which substantiates its high quality, corroborating with TIPL and PDS studies, and also justifies the high photovoltage delivered by $\mathrm{FAPbBr}_{3}$ devices. TRPL also reveals that charge carriers have extremely short life time ( $\tau_{1}=2 \mathrm{~ns}$ ) when $\mathrm{FAPbBr}_{3}$ is deposited onto Li-treated $\mathrm{TiO}_{2}$, which illustrates more efficient injection of electrons from the conduction band of $\mathrm{FAPbBr}_{3}$ into that of mesoporous $\mathrm{TiO}_{2}$. Compared to electron injection into the conduction band of pristine $\mathrm{TiO}_{2}$, the injection of holes from the valence band of $\mathrm{FAPbBr}_{3}$ into the highest occupied molecular orbital of spiro-OMeTAD is faster. 

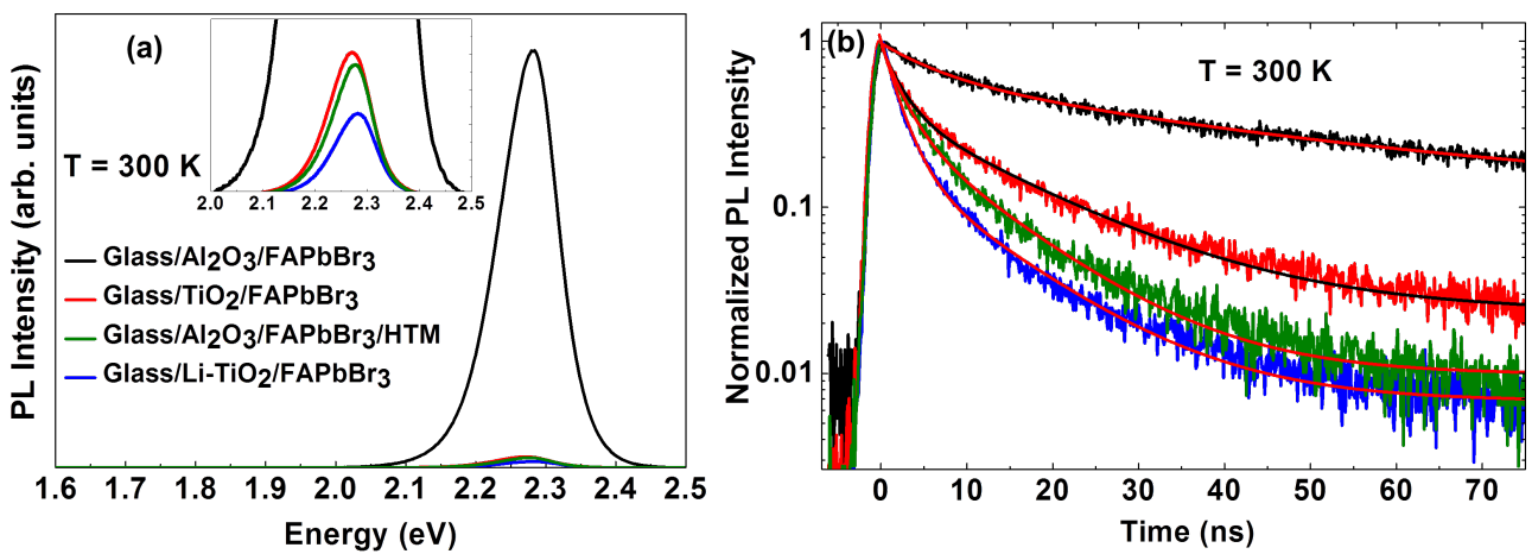

Figure 5. Photoluminescence studies of $\mathrm{FAPbBr}_{3}$ perovskite films deposited on different substrates recorded at $300 \mathrm{~K}$ : (a) time-integrated photoluminescence spectra (Inset shows zoomed TIPL spectra), and (b) time-resolved photoluminescence spectra plotted on a semi-logarithmic scale. Solid lines present the fitting curves obtained with two-exponential decay model. TRPL spectra were measured at a wavelength near band gap that yields maximum PL signal upon exciting perovskite samples at $420 \mathrm{~nm}$. (The color code for Figure $\mathbf{a}$ and $\mathbf{b}$ legends is same)

Table 1. Summarized emission lifetime and corresponding intensities for various perovskite samples obtained by using two exponential decay model.

\begin{tabular}{|c|c|c|c|c|}
\hline Sample & $\boldsymbol{\tau}_{\mathbf{1}}(\mathbf{n s})$ & $\mathbf{A ( \% )}$ & $\boldsymbol{\tau}_{\mathbf{2}}$ & $\mathbf{A ( \% )}$ \\
\hline Glass $/ \mathrm{Al}_{2} \mathrm{O}_{3} / \mathrm{FAPbBr}_{3}$ & 41 & 55 & 6.4 & 45 \\
\hline $\mathrm{Glass} / \mathrm{TiO}_{2} / \mathrm{FAPbBr}_{3}$ & 2.3 & 60 & 15 & 40 \\
\hline Glass $/ \mathrm{Al}_{2} \mathrm{O}_{3} / \mathrm{FAPbBr}_{3} / \mathrm{HTM}$ & 2.2 & 67 & 11 & 33 \\
\hline Glass $/ \mathrm{Li}$-treated $\mathrm{TiO}_{2} / \mathrm{FAPbBr}_{3}$ & 2 & 88 & 11 & 12 \\
\hline
\end{tabular}

\section{Stability of $\mathrm{FAPbBr}_{3}$ devices}

The extrinsic and intrinsic instabilities under operational conditions impede the practical application of perovskite solar cells. Apart from the sensitivity of organic components, the extrinsic instability arises majorly from the hygroscopic nature of perovskite material, whereas the intrinsic instability can be associated with the structure of the absorber material. ${ }^{26} \mathrm{We}$ examined the photo- and humidity stability of unsealed $\mathrm{FAPbBr}_{3}$ devices by measuring the photovoltaic efficiency at maximum power point as a function of time under three different conditions. Firstly, we evaluated the photostability of $\mathrm{FAPbBr}_{3}$ device under flowing nitrogen 
atmosphere. The $\mathrm{FAPbBr}_{3}$ perovskite solar cell displayed an exceptional stability over a period of $150 \mathrm{~h}$ under full sun illumination (Figure 6a). In most of the stability studies presented so far, the perovskite devices were either encapsulated or processed under inert conditions. But for long-term applications, it is very important that perovskite solar cell must survive under aggressive conditions. Therefore, we examined the photovoltaic performance of unsealed $\mathrm{FAPbBr}_{3}$ devices under relatively dry conditions (Humidity 10\%) Evidently, the device exhibited better stability (in fact improved with time) as compared to those examined under inert conditions. Apparently, the device performance remained robust for $150 \mathrm{~h}$ at a maximum power point under continuous full sun illumination. Finally, to examine the vulnerability of perovskites to moisture, we exposed the $\mathrm{FAPbBr}_{3}$ devices to relative high humidity of $>60 \%$ at maximum power point under continuous full sun illumination. Surprisingly, no severe degradation was observed for $150 \mathrm{~h}$ demonstrating a very good stability of $\mathrm{FAPbBr}_{3}$. To the best of our knowledge, this is the first ever demonstration of an unprecedented stability for bromide based perovskites devices yielding a high photovoltage examined under extreme and operational conditions. Earlier Kulbak et al. examined the stability of photocurrent density at maximum power point for cesium lead bromide $\left(\mathrm{CsPbBr}_{3}\right)$ perovskite based devices ${ }^{27}$, however, authors observed a decay of $13 \%$ in the current density merely during 5 hrs of illumination. Furthermore, we also evaluated the device performance while storing unsealed $\mathrm{FAPbBr}_{3}$ perovskite devices under ambient conditions (Figure 6b). After recording the current-voltage curves at a regular interval of 6 days for over 6 months, the devices showed no significant decay and retained $90 \%$ of their efficiency.

As mentioned before, long-term stability under operational conditions is one of the main challenges that preclude the commercialization of perovskite solar cells. The extraordinary stability and reproducibility exhibited by these $\mathrm{FAPbBr}_{3}$ devices yielding high $V_{\text {oc }}$ under extreme conditions over longer periods of time is commendable. There are reports addressing the stability issue by controlling the morphology, encapsulation of active layers, but it is extremely important to identify intrinsically stable perovskites crystal structure for practical applications. ${ }^{28} \mathrm{We}$ attribute the exceptional stability to the low polarity of the FA cation and inherent structure of the $\mathrm{FAPbBr}_{3}$ perovskite material itself. The homogeneous distribution of the electron-density over the C-N bonds results in the resonance stabilization of the FA cation structure, ${ }^{29,30}$ which eventually brings down it affinity towards moisture and other reactive species. 

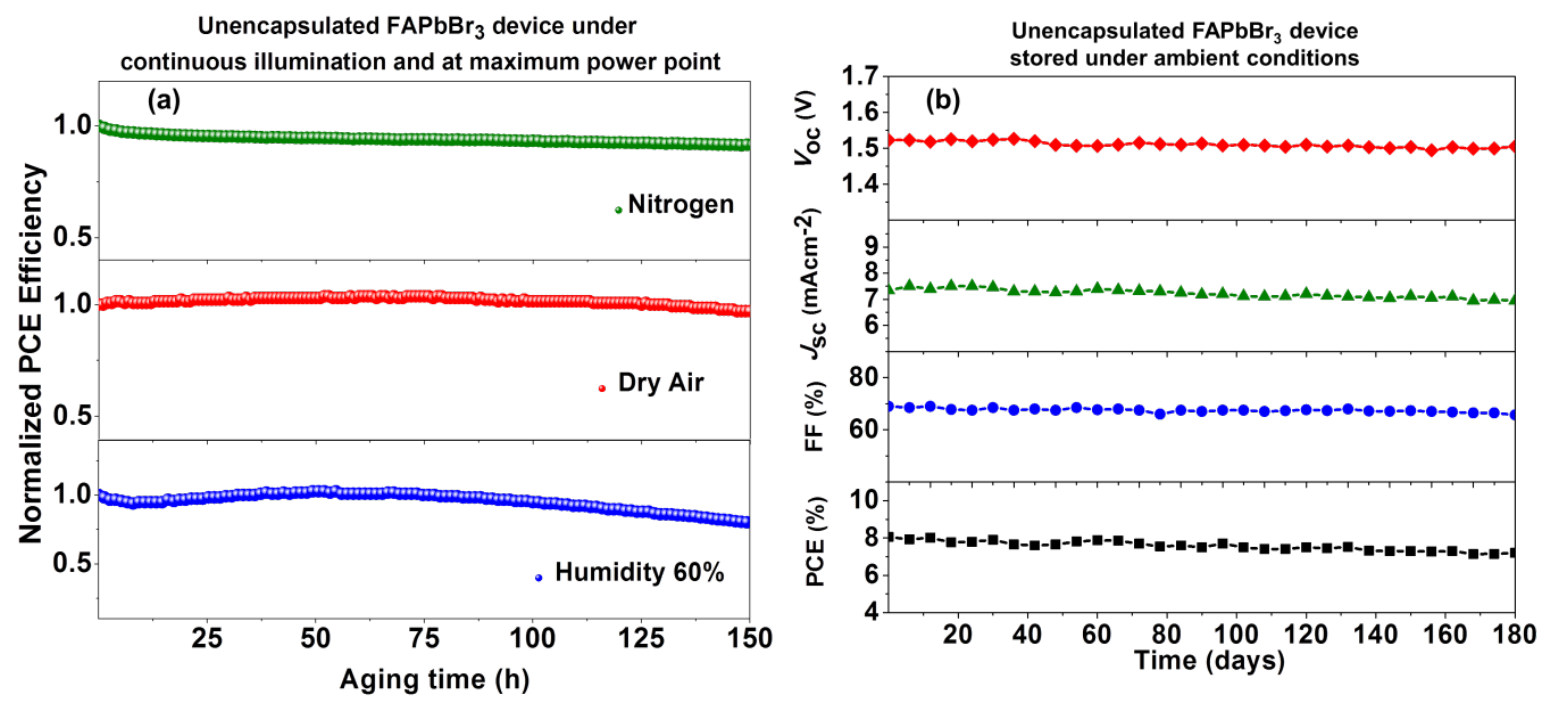

Figure 6. Operational stability of unencapsulated devices. a. Maximum power point tracking under continuous illumination using three different conditions and $\mathbf{b}$. Evolution of photovoltaic performance parameters of device stored in ambient conditions as a function of time. The cell parameters $\left(V_{\mathrm{oc}}, J_{\mathrm{sc}}, \mathrm{FF}\right.$, and PCE) were collected under AM1.5 simulated sun light at a regular interval of 6 days.

\section{Conclusion}

In conclusion, we report on the fabrication of highly stable $\mathrm{FAPbBr}_{3}$ perovskite devices exhibiting an outstanding photovoltage of $1.53 \mathrm{~V}$ and a high power conversion efficiency of 8.2 \% under AM1.5 simulated sun light. Various factors leading to improved photovoltaic performance were unraveled using a combination of techniques including photothermal deflection absorption spectroscopy, transient photovoltage measurements, time-integrated and time-resolved photoluminescence. Photothermal deflection spectroscopy confirmed the lower sub-bandgap absorption level for Li-treated titania in comparison to pristine titania which results in lower energetic disorder within the $\mathrm{TiO}_{2}$ /perovskite interface. The transient photovoltage decay measurements brought out improved electron lifetime and less density of traps which improved the photovoltage of $\mathrm{FAPbBr}_{3}$ perovskite devices. The time-integrated and timeresolved photoluminescence illustrates relatively more efficient injection of electrons from the conduction band of $\mathrm{FAPbBr}_{3}$ into that of Li-treated mesoporous $\mathrm{TiO}_{2}$. Importantly, the advantage of such $\mathrm{FAPbBr}_{3}$ solar cells was further demonstrated by carrying out stability tests at maximum 
power point under extreme conditions. The un-encapsulation devices displayed unprecedented photostability of over $150 \mathrm{~h}$ under continuous sunlight illumination and outstanding stability upon long-term aging $(4000 \mathrm{~h})$. We attributed the enhanced photo-and humidity stability to the less polarity of FA cation and inherent structure of $\mathrm{FAPbBr}_{3}$ perovskite material. On the basis of our current investigation, we strive to further improve the device performance by optimizing the device architecture and exploring HTM with deeper the highest occupied molecular orbital.

\section{Experimental section}

All materials were purchased from Sigma-Aldrich were used as received unless stated otherwise.

\section{Preparation of $\mathrm{TiO}_{2}$ photoanode}

Fluorine-doped tin oxide (FTO)-glass substrates (TCO glass, NSG 10, Nippon sheet glass, Japan) were cleaned by ultrasonication in Hellmanex (2\%, deionized water), rinsed thoroughly with de-ionized water and ethanol, and then treated in oxygen plasma for $15 \mathrm{~min}$. A compact layer of $\mathrm{TiO}_{2}$ of ca $30 \mathrm{~nm}$ was subsequently deposited via spray pyrolysis at $450{ }^{\circ} \mathrm{C}$ using a commercial titanium diisopropoxide bis(acetylacetonate) solution (75\% in 2-propanol, SigmaAldrich) diluted in anhydrous ethanol (1:9, volume ratio) as precursor and oxygen as carrier gas. A mesoporous $\mathrm{TiO}_{2}$ layer was then deposited by spin coating a diluted paste (1:3.5 wt. ratio) (Dyesol 30NRD: ethanol) (5000 rpm, acceleration $2000 \mathrm{rpm}$ for $30 \mathrm{~s}$ ) onto the substrate containing $\mathrm{TiO}_{2}$ compact layer. This was followed by a series of sintering steps (325 ${ }^{\circ} \mathrm{C}$ for 5 min with 15 min ramp time, $375^{\circ} \mathrm{C}$ for 5 min with 5 min ramp time, $450{ }^{\circ} \mathrm{C}$ for 30 min with 5 min ramp time) in dry air. For $\mathrm{Li}$ treatment of mesoporous $\mathrm{TiO}_{2}$ photoanode $200 \mu \mathrm{L}$ of LiTFSI solution in acetonitrile (20mg/mL, freshly prepared in argon atmosphere) was spin coated (3000 rpm, acceleration $2000 \mathrm{rpm}$ for $20 \mathrm{~s}$ ) after a loading time of $10 \mathrm{~s}$. Thereafter, Li treated and untreated substrates were subjected to second sintering step at $450{ }^{\circ} \mathrm{C}$ for $30 \mathrm{~min}$. The substrates were transferred in a dry box (humidity< 1\%) after cooling down to $200{ }^{\circ} \mathrm{C}$ for perovskite deposition. 250 nm-thick $\mathrm{Al}_{2} \mathrm{O}_{3}$ mesoporous layer composed of $30 \mathrm{~nm} \mathrm{Al}_{2} \mathrm{O}_{3}$ was deposited on non-conducting glass substrate by spin coating diluted paste (1:3.5 wt. ratio) (5000 rpm, 
acceleration $2000 \mathrm{rpm}$ for $30 \mathrm{~s}$ ). After sintering at $450{ }^{\circ} \mathrm{C}$ for $30 \mathrm{~min}$ with $10 \mathrm{~min}$ ramp time, mesoporous $\mathrm{Al}_{2} \mathrm{O}_{3}$ films were obtained.

\section{Device fabrication}

$\mathrm{FAPbBr}_{3}$ films were deposited using two-step sequential deposition method. 1.2 $\mathrm{M} \mathrm{PbBr}_{2}$ (TCI 99\%) precursor solutions were prepared in $N, N$-dimethylformamide (DMF, Acros Organics) and dimethylsulphoxide (DMSO, Acros Organics) by constant stirring at $60{ }^{\circ} \mathrm{C}$ for 30 minutes. $\mathrm{PbBr}_{2}$ (DMF+DMSO in 1:1 volume ratio) solution was spin-coated onto the mesoporous $\mathrm{TiO}_{2}$ films at $3000 \mathrm{rpm}$ for $30 \mathrm{~s}$. This was followed by annealing the films at $80{ }^{\circ} \mathrm{C}$ for $15 \mathrm{~min}$. Then the films were dipped in $\mathrm{CH}\left(\mathrm{NH}_{2}\right)_{2}$ solution (Dyesol, $50 \mathrm{mM}$ ) in isopropanol for $5 \mathrm{~min}$ at $60{ }^{\circ} \mathrm{C}$, rinsed with 2-propanol for $5 \mathrm{~s}$ and dried at $80^{\circ} \mathrm{C}$ for $30 \mathrm{~min} .72 .3 \mathrm{mg}$ Spiro-OMeTAD (2,2',7,7’tetrakis( $N, N$-di-p-methoxyphenylamine)-9,9-spirobifluorene) was dissolved in $1 \mathrm{~mL}$ of chlorobenzene, to which $28.8 \mu \mathrm{L}$ 4-tert-butylpyridine, $17.5 \mu \mathrm{L}$ of a stock solution of

$520 \mathrm{mg} \mathrm{mL}^{-1}$ bis(trifluoromethylsulphonyl)imide in acetonitrile, and $29 \mu \mathrm{L}$ of a stock solution of $300 \mathrm{mg} \mathrm{mL}^{-1}$ tris(2-(1H-pyrazol-1-yl)-4-tert-butylpyridine)cobalt(III)

bis(trifluoromethylsulphonyl)imide in acetonitrile were added. The device fabrication was carried out under controlled atmospheric conditions with humidity $<1 \%$. Finally, device fabrication was completed by thermally evaporating $70 \mathrm{~nm}$ of gold layer as a back contact.

\section{Characterization}

XRD data were collected on a Bruker Advance D8 X-ray diffractometer with a graphite monochromator, using $\mathrm{Cu}-\mathrm{K} \alpha$ radiation, at a scanning rate of $0.5^{\circ} \mathrm{min}^{-1}$. Field-emission scanning electron microscope (FESEM, Merlin) was employed to analyze the morphology of the samples. An electron beam accelerated to $3 \mathrm{kV}$ was used with an in-lens detector.

Device characterization: The current-voltage $(\mathrm{J}-\mathrm{V})$ characteristics of the perovskite devices were recorded with a digital source meter (Keithley model 2400, USA). A 450 W xenon lamp (Oriel, USA) was used as the light source for photovoltaic $(J-V)$ measurements. The spectral output of the lamp was filtered using a Schott K113 Tempax sunlight filter (Präzisions Glas \& Optik GmbH, Germany) to reduce the mismatch between the simulated and actual solar spectrum to less than $2 \%$. The photo-active area of $0.16 \mathrm{~cm}^{2}$ was defined using a dark-coloured metal mask. 
Incident photon-to-current conversion efficiency (IPCE) measurements were made using a 300 W xenon light source (ILC Technology, USA). A double-monochromator spectrometer (Gemini180, Jobin Yvon Ltd., UK) was used to select and increment the wavelength of the radiation impinging on the cells. The incident monochromatic light was passed through a chopper running at $1 \mathrm{~Hz}$ and the on/off ratio was measured by an operational amplifier. This was superimposed on a white light bias corresponding to an intensity of $10 \mathrm{~mW} / \mathrm{cm}^{2}$.

Charge extraction and photovoltage transient analysis: Transient photovoltage measurements were performed in quasi steady-state conditions. The devices were illuminated by a continuous calibrated LED light (array of 9 Luxeonstar 5650K) on which a small uniform optical perturbation from a Green LED (Luxeonstar 655nm) was super imposed. Perturbations were never exceeding $4 \mathrm{mV}$ (for transient photovoltage) Devices were held under high load (1MOhm) for transient photovoltage. Fast transients $(>1 \mathrm{kHz})$ have been recorded on a DPO7000C oscilloscope with AC coupling. Decay signals were fitted by monoexponentials. Charge extraction at open circuit has been performed by holding the device under a high load using an open MOSFET transistor (BS170) and constant bias light illumination. After 1 second of illumination, the light was turned off and the transistor closed. A variable small load resistor was placed between the transistor and the working electrode (grounded) to record the current transient. It was ensured that the IR drop across the resistor never caused the transistor to open during extraction. Gate voltage was $8 \mathrm{~V}$. The extraction current was integrated on an oscilloscope (DPO7000C) from 0.1us to 100ms to yield the extracted charge density.

Photothermal deflection spectroscopy (PDS): is a scatter-free surface sensitive absorption measurement capable of measuring 5-6 orders of magnitude weaker absorbance than the band edge absorption. For the measurements, a monochromatic Pump light beam is shined on the sample (film on Quartz substrate), which on absorption produces a thermal gradient near the sample surface via non-radiative relaxation induced heating. This results in a refractive index gradient in the area surrounding the sample surface. This refractive index gradient is further enhanced by immersing the sample in an inert liquid FC-72 Fluorinert ${ }^{\circledR}$ (3M Company) which has a high refractive index change per unit change in temperature. A fixed wavelength CW laser Probe beam is passed through this refractive index gradient producing a deflection proportional to the absorbed light at that particular wavelength, which is detected by a photo-diode and lock- 
in amplifier combination. Scanning through different wavelengths gives us the complete absorption spectra. Because this technique makes use of the non-radiative relaxation processes in the sample, makes it immune to optical effects like interference and scattering.

Photoluminescence spectroscopy: Time integrated photoluminescence (PL) and time-resolved photoluminescence (TRPL) experiments were performed by exciting the $\mathrm{FAPbBr}_{3}$ samples deposited onto mesoporous $\mathrm{Al}_{2} \mathrm{O}_{3}$ layer (with and without spiro-OMeTAD) and onto mesoporous $\mathrm{TiO}_{2}$ (with and without Li-treatment) at $420 \mathrm{~nm}$. All the samples were excited from the perovskite side under ambient conditions. To record photoluminescence spectra and PL decay kinetics, the samples were excited by the second harmonic of a picosecond mode-locked Ti:Sapphire laser ( $80.5 \mathrm{MHz}$ ). The excitation beam is focused on the sample by means of a 90$\mathrm{mm}$-focal that allows for an excitation spot diameter of around $100 \mu \mathrm{m}$. The average power was kept in the range 1-10 $\mathrm{mW}$, which corresponds to 0.05 to $0.5 \mu \mathrm{J} / \mathrm{cm}^{2}$ per pulse. The collected PL spectra were spectrally and temporally analyzed using a 32-cm-focal length monochromator equipped with a charge-coupled device (CCD) which has a spectral resolution of $>1 \mathrm{meV}$ and the streak camera with a temporal resolution of $\sim 5 \mathrm{ps}$, respectively. 


\section{References}

1 Mitzi, D. B. Solution-Processed Inorganic Semiconductors. J. Mater. Chem. 2004, 14, 2355-2365.

${ }^{2}$ H.-S. Kim , C.-R. Lee , J.-H. Im , K.-B. Lee , T. Moehl , A. Marchioro , S.-J. Moon, R. Humphry-Baker, J.-H. Yum , J. E. Moser, M. Grätzel , N.-G. Park , Sci. Rep. 2012 , 2 , 591.

${ }^{3}$ A. Kojima , K. Teshima , Y. Shirai , T. Miyasaka , J. Am. Chem. Soc. 2009 , 131 , 6050 .

${ }^{4}$ B. O’Regan , M. Grätzel , Nature 1991 , 353 , 737 .

${ }^{5}$ Aravind Kumar Chandiran, Mojtaba Abdi-Jalebi, Aswani Yella, M Ibrahim Dar, Chenyi Yi, Srinivasrao A Shivashankar, Mohammad K Nazeeruddin, Michael Grätzel, Nano Lett., 2014, 14 (3), pp 1190-1195

${ }^{6}$ Mukes Kapilashrami, Yanfeng Zhang, Yi-Sheng Liu, Anders Hagfeldt, and Jinghua Guo Chem. Rev. 2014, 114, 9662-9707

${ }^{7}$ P. Qin, A. L. Domanski, A. K. Chandiran, R. Berger, H.-J. Butt, M. I. Dar, T. Moehl, N. Tetreault, P. Gao, S. Ahmad, M. K. Nazeeruddin, M. Gratzel, Yttrium-substituted nanocrystalline $\mathrm{TiO} 2$ photoanodes for perovskite based heterojunction solar cells. Nanoscale $\mathbf{6}$, $1508-1514$

${ }^{8}$ Bart Roose, Karl C. Gödel, Sandeep Pathak, Aditya Sadhanala, Juan Pablo Correa Baena, Bodo D. Wilts , Henry J. Snaith, Ulrich Wiesner , Michael Grätzel, Ullrich Steiner, and Antonio Abate Adv. Energy Mater. 2016, 6, 1501868

${ }^{9}$ Aravind Kumar Chandiran, Frédéric Sauvage, Lioz Etgar, and Michael Graetzel J. Phys. Chem. C, 2011, 115 (18), pp 9232-9240

10 J. Burschka , N. Pellet, S.-J. Moon , R. Humphry-Baker , P. Gao , M. K. Nazeeruddin , M. Grätzel , Nature 2013 , 499 , 316 .

${ }^{11}$ N. J. Jeon , J. H. Noh, W. S. Yang , Y. C. Kim , S. Ryu , J. Seo , S. I. Seok , Nature 2015 , 517,476 .

${ }^{12}$ N. J. Jeon , J. H. Noh , Y. C. Kim , W. S. Yang , S. Ryu , S. I. Seok , Nat. Mater. 2014 , 13 , 897.

${ }^{13}$ M. Schreier, L. Curvat, F. Giordano, L. Steier, A. Abate, S.M. Zakeeruddin, J. Luo, M. Mayer, M. Grätzel "Efficient photosynthesis of carbon monoxide from CO2 using perovskite photovoltaics" Nature Communications 6, 7326 (2015) 
14 Edri, E.; Kirmayer, S.; Kulbak, M.; Hodes, G.; Cahen, D. Chloride Inclusion and Hole Transport Material Doping to Improve Methyl Ammonium Lead Bromide Perovskite-Based High Open- Circuit Voltage Solar Cells. J. Phys. Chem. Lett. 2014, 5, 429-433.

${ }^{15}$ Heo, J. H.; Song, D. H.; Im, S. H. Planar CH3NH3PbBr3 Hybrid Solar Cells with 10.4\% Power Conversion Efficiency, Fabricated by Controlled Crystallization in the Spin-Coating Process. Adv. Mater. 2014, 26, 8179-8183.

${ }^{16}$ Ryu, S.; Noh, J. H.; Jeon, N. J.; Chan Kim, Y.; Yang, W. S.; Seo, J.; Seok, S. I. Voltage Output of Efficient Perovskite Solar Cells with High Open-Circuit Voltage and Fill Factor. Energy Environ. Sci. 2014, 7, 2614-2618.

17 Frost, J. M.; Butler, K. T.; Brivio, F.; Hendon, C. H.; van Schilfgaarde, M.; Walsh, A. Atomistic Origins of High-Performance in Hybrid Halide Perovskite Solar Cells. Nano Lett. 2014, 14, 2584-90.

18 J. Phys. Chem. Lett. 2016, 7, 167-172

19 Heo, J. H. et al. Hysteresis-less mesoscopic CH3NH3PbI3 perovskite hybrid solar cells by introduction of Li-treated TiO2 electrode. Nano Energy 15, 530-539 (2015).

${ }^{20}$ Giordano, F. et al. Enhanced electronic properties in mesoporous TiO2 via lithium doping for high-efficiency perovskite solar cells. Nat. Commun. 7:10379 doi: 10.1038/ncomms10379 (2016).

${ }^{21}$ Giordano, F. et al. Enhanced electronic properties in mesoporous TiO2 via lithium doping for high-efficiency perovskite solar cells. Nat. Commun. 7:10379 doi: 10.1038/ncomms10379 (2016).

${ }^{22}$ Arora, N.; Dar, M. I.; Hezam, M.; Tress, W.; Jacopin, G.; Moehl, T.; Gao, P.; Aldwayyan, A. S.; Deveaud, B.; Grätzel, M.; et al. Photovoltaic and Amplified Spontaneous Emission Studies of High- Quality Formamidinium Lead Bromide Perovskite Films. Adv. Funct. Mater. 2016, 26, $2846-2854$.

${ }^{23}$ Arora, N.; Dar, M. I.; Hezam, M.; Tress, W.; Jacopin, G.; Moehl, T.; Gao, P.; Aldwayyan, A. S.; Deveaud, B.; Grätzel, M.; et al. Photovoltaic and Amplified Spontaneous Emission Studies of High- Quality Formamidinium Lead Bromide Perovskite Films. Adv. Funct. Mater. 2016, 26, 2846-2854.

${ }^{24}$ Hanusch, F. C.; Wiesenmayer, E.; Mankel, E.; Binek, A.; Angloher, P.; Fraunhofer, C.; Giesbrecht, N.; Feckl, J. M.; Jaegermann, W.; Johrendt, D.; et al. Efficient Planar Heterojunction 
Perovskite Solar Cells Based on Formamidinium Lead Bromide. J. Phys. Chem. Lett. 2014, 5, 2791-2795.

${ }^{25}$ Abdi-Jalebi, M. et al. Impact of Monovalent Cation Halide Additives on the Structural and Optoelectronic Properties of CH3NH3PbI3 Perovskite. Adv. Energy Mater. 201502472, (2016).

${ }^{26}$ Adv. Energy Mater. 2015, 5, 1501310

${ }^{27}$ Michael Kulbak, Satyajit Gupta,Nir Kedem, Igal Levine, Tatyana Bendikov,Gary Hodes,, and David Cahen J. Phys. Chem. Lett. 2016, 7, 167-172

${ }^{28}$ Bert Conings, Jeroen Drijkoningen , Nicolas Gauquelin , Aslihan Babayigit , Jan D’Haen , Lien D’Olieslaeger, Anitha Ethirajan , Jo Verbeeck , Jean Manca, Edoardo Mosconi , Filippo De Angelis , and Hans-Gerd Boyen Adv. Energy Mater. 2015, 5, 1500477

${ }^{29}$ D. B. Mitzi , K. Liang, J. Solid State Chem. 1997 , 134 , 376 .

${ }^{30}$ Frost, J. M.; Butler, K. T.; Brivio, F.; Hendon, C. H.; van Schilfgaarde, M.; Walsh, A. Atomistic Origins of High-Performance in Hybrid Halide Perovskite Solar Cells. Nano Lett. 2014, 14, 2584-90. 